\title{
Thermal Modeling of Optical Power Absorption in Moving Multilayer Thin Films
}

\author{
W.A. Challener* and A. Itagi
}

Seagate Technology, Pittsburgh, PA 15222, USA

\begin{abstract}
A technique for computing the thermal profile in a multilayer moving medium is described. This technique is particularly suitable for studying the near field optical/thermal interplay in hybrid optical/magnetic recording because the boundaries of the computation space are effectively removed from the optical source. It is shown that a three layer medium can be designed with a thermal time constant which is suitable for high recording data rates and that minimizes the thermal bloom from motion of the medium with respect to the optical spot. However, the thermal spot is much larger than the optical spot which leads to a reduced storage density.
\end{abstract}

\section{INTRODUCTION}

Numerical techniques for efficiently computing the temperature rise [1-3] in a multilayer thin film stack [4-6] due to a focused laser beam have been developed for a variety of applications such as recordable optical data storage. The focused optical spot size for current optical storage products ranges from $\sim 0.88 \mu \mathrm{m}$ for CD's at a wavelength of $780 \mathrm{~nm}$ to $0.24 \mu \mathrm{m}$ for Blu-Ray disks at a wavelength of $405 \mathrm{~nm}$. When the optical spot is much larger than or comparable to the thickness of the thin film stack, the heat sink/reflector layer in the disk ensures that the dominant direction for heat flow is perpendicular to the thin films. As a result, the thermal boundary conditions in the lateral direction are not difficult to handle with simple approximations, such as a quadratic temperature dependence at the boundary [4].

Future optical data storage products may require near field optics to achieve larger storage densities. Magnetic hard discs may also incorporate a hybrid optical/magnetic technology called "heat assisted magnetic recording" (HAMR) using near field optics to transfer the optical energy into the recording medium in a highly localized spot smaller than the total thickness of the film stack $[7,8]$. In such a case, the lateral heat flow can become as important as the perpendicular heat flow and thermal boundary conditions must be carefully applied.

The advantages of the alternate direction implicit (ADI) technique for thermal modeling of optical data storage media have been described by Mansuripur et al. [4,5] They developed the ADI equations for a cylindrical coordinate system and a circularly symmetric optical spot in both the stationary and moving frame of reference. The ADI equations were subsequently applied by Peng et al. [6] to a stationary 3D Cartesian coordinate system to investigate the amorphization and crystallization dynamics of optical phase change media.

*Address correspondence to this author at the Seagate Technology, 1251 Waterfront Place, Pittsburgh, PA 15222, USA; Tel. 412-918-7197; Fax: 412-918-7010; E-mail: william.a.challener@seagate.com
Itagi [9] extended the ADI equations to include variable layer thicknesses, thereby enabling a substantial decrease in computation time for film stacks with thick layers. He also derived the ADI equations for the moving frame in a cylindrical coordinate system [10]. In this paper we present the ADI equations for a Cartesian coordinate system in the moving frame of reference and a method for accurately handling the lateral thermal boundary conditions in a multilayer film stack, and then apply these results to a HAMR recording medium.

\section{BASIC THEORY}

The Fourier heat conduction equation is

$$
C(\overrightarrow{\mathbf{r}}, t) \frac{\partial}{\partial t} T(\overrightarrow{\mathbf{r}}, t)=\nabla \cdot[\tilde{\mathbf{k}}(\overrightarrow{\mathbf{r}}, t) \nabla T(\overrightarrow{\mathbf{r}}, t)]+g(\overrightarrow{\mathbf{r}}, t)
$$

where $\mathrm{C}$ is the heat capacity, $\tilde{\mathbf{k}}$ is the thermal conductivity tensor, $\mathrm{g}$ is the input power, $\mathrm{t}$ is the time, and $\mathrm{T}$ is the temperature. Eq. (1) can be implemented numerically by subdividing the region of interest into many smaller cells to approximate the spatial gradients, and stepping the time in discrete increments. In the explicit method, the temperature at a specific point in the cell space at time $t=n+1$ is completely determined by the temperatures within the cell space for the previous time step, $\mathrm{t}=\mathrm{n}$.

$$
C \frac{\left(T^{n+1}-T^{n}\right)}{\Delta t}=\nabla \cdot\left(\tilde{\mathbf{k}} \nabla T^{n}\right)+g .
$$

There is only one unknown variable, $\mathrm{T}^{\mathrm{n}+1}(\mathrm{i}, \mathrm{j}, \mathrm{k})$, at each point in the cell space and time step. However, there is a constraint on the size of the time step to ensure numerical stability of the calculation. It is generally necessary to choose very small time steps, and as a result, the simple explicit technique is not suitable for many problems of practical interest.

The simplest implicit method for solving the heat conduction equation rewrites Eq. (1) as

$$
C \frac{\left(T^{n+1}-T^{n}\right)}{\Delta t}=\nabla \cdot\left(\tilde{\mathbf{k}} \nabla T^{n+1}\right)+g .
$$


In this case the change in temperature between time step $\mathrm{n}+1$ and time step $\mathrm{n}$ is expressed in terms of the temperatures at time step $n+1$. There are seven unknown variables in this equation, the temperatures at $\mathrm{t}=\mathrm{n}+1$ at point $(\mathrm{i}, \mathrm{j}, \mathrm{k})$ and its six neighbors, making the solution of the set of equations in cell space much more complex and time consuming, although this technique has the advantage of unconditional stability.

The Crank-Nicolson $(\mathrm{CN})$ technique is an average of the implicit and explicit techniques. The $\mathrm{CN}$ equation is

$$
C \frac{\left(T^{n+1}-T^{n}\right)}{\Delta t}=\nabla \cdot \tilde{\mathbf{k}} \nabla \frac{\left(T^{n+1}+T^{n}\right)}{2}+g \text {. }
$$

This technique is also unconditionally stable and the accuracy is second order in $\Delta t$, which is greater than either that of the simple implicit or simple explicit techniques. However, there are still seven unknown quantities in this equation at each point and time step making it computationally intensive to solve directly.

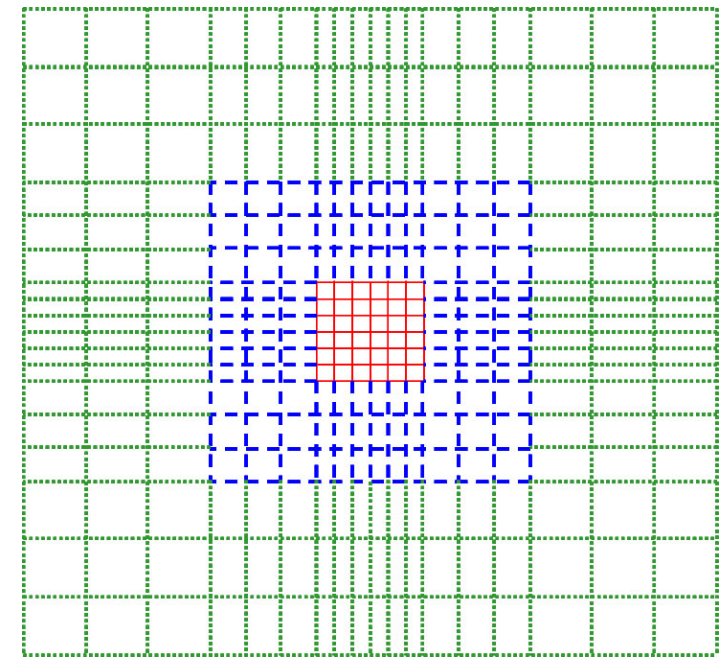

Fig. (1). Cell space in the horizontal plane with extended boundaries. The central red region is the standard computational space with uniform cell sizes. This region is ringed by multiple additional regions with varying cell sizes so that the final cell space is orders of magnitude larger than the central region.

Douglas and Gunn [7] developed an ADI algorithm for the $\mathrm{CN}$ equation that maintains its unconditional stability and numerical accuracy but greatly reduces the computation time. In their technique Eq. (5) at each time step is subdivided into two or more equations with fewer unknowns that can be solved sequentially to determine the change in temperature. The general approach for the stationary coordinate system is discussed in Ref. [3]. In this paper we develop the ADI equations for the moving frame of reference in the 3D Cartesian coordinate system.

A problem with the numerical solution to the heat flow equation is handling the boundaries of the finite computation space appropriately. Ideally the boundaries of the computation space are placed far enough from the region of interest that the boundaries have no effect on the thermal calculation within the time interval of interest. In some cases, however, this approach requires unreasonably large computation spaces. In particular, we have found that for modeling heat flow in HAMR media due to optical energy delivered to the medium in a tightly confined spot in the near field, the effects of boundaries in a finite computation space must be carefully handled. In the next section we develop a method of extending the boundaries by variable cell sizes to completely remove boundary effects.

\section{EXTENDED BOUNDARIES}

For convenience, the vertical direction will be defined to be perpendicular to the plane of the films. To implement extended boundaries the ADI equations are first modified to handle a variable cell size in the lateral direction. In the region of interest in the center of the computation space where the optical power is delivered to the films, the cells are discretized laterally with a fine grid. Outside of this center region are rings of cells with successively larger lateral dimensions as shown in Fig. (1). Four sets of rings that are each five cells thick have been chosen for the results described in section 4, and in each ring the lateral dimension(s) are chosen to be ten times greater than that of the ring immediately inside it. For example, if the central cell space region is 500 by 500 cells with $(20 \mathrm{~nm})^{2}$ cell areas corresponding to a $10 \times$ $10 \mu \mathrm{m}$ cell space, the extended cell space has $540 \times 540$ cells covering a $2 \times 2 \mathrm{~mm}$ area. Thus the cell space boundaries are too far from the central heat source to effect the calculation for any time interval that is practical to compute.

The equations for the numerical calculation are obtained using the approach of Itagi [10]. Following the notation in that reference, a specific point in the cell space is considered with coordinates $(\mathrm{i}, \mathrm{j}, \mathrm{k})$ as shown in Fig. (2). The points are located at the corners of the cells and the temperatures are defined at each point. The thermal conductivities are defined on the cell edges between points while the heat capacities are defined for each cell volume. Some cells in the computation space are surrounded by other cells of the same material and cell dimensions. The thermal conductivity, heat capacity, and cell dimensions associated with the points on the corners of these cells are then clearly defined. On the other hand, some points occur at the boundary between layers of different materials, and some points occur at the boundary between cells of different dimensions as shown in Fig. (2). To compute the temperature at these points with the ADI equations some additional definitions are helpful.

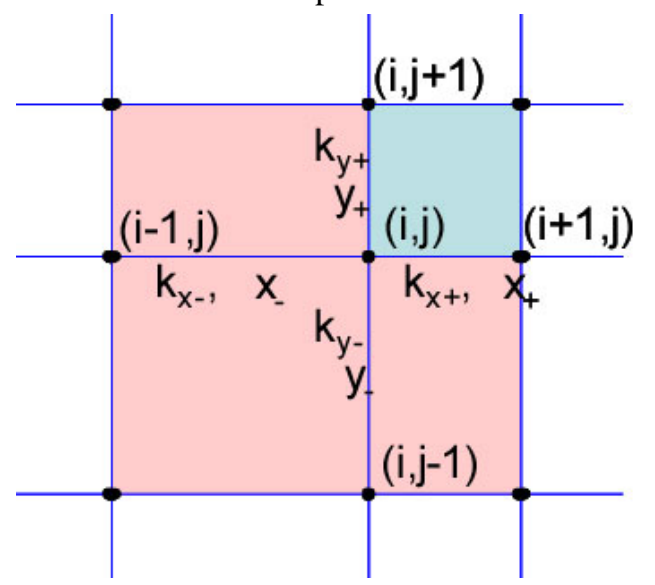

Fig. (2). Illustration of parameter definitions at boundaries of nonuniform cells within a thin film layer. 


$$
\begin{aligned}
& \Delta x_{+} \equiv x_{j+1}-x_{j} \\
& \Delta y_{+} \equiv y_{j+1}-y_{j} \\
& \Delta z_{+} \equiv z_{j+1}-z_{j} \\
& \Delta x_{-} \equiv x_{j}-x_{j-1} \\
& \Delta y_{-} \equiv y_{j}-y_{j-1} \\
& \Delta z_{-} \equiv z_{j}-z_{j-1} \\
& \Delta x \equiv 0.5\left(\Delta x_{+}+\Delta x_{-}\right) \\
& \Delta y \equiv 0.5\left(\Delta y_{+}+\Delta y_{-}\right)
\end{aligned}
$$

and

$$
\Delta z \equiv 0.5\left(\Delta z_{+}+\Delta z_{-}\right) .
$$

The heat capacity assigned to point $(\mathrm{i}, \mathrm{j}, \mathrm{k})$ is an average of the heat capacity for the material in the layer above the point, $\mathrm{C}_{+}$, and the layer below the point, $\mathrm{C}_{-}$,

$$
C_{k} \equiv \frac{\Delta z_{+} C_{+}+\Delta z_{-} C_{-}}{\Delta z_{+}+\Delta z_{-}} .
$$

Because the lateral dimensions for the cells in the upper layer are the same as the cells in the lower layer on either side of the point, this is equivalent to a volumetric average of the heat capacity.

The thermal conductivity above and below the point is that for the layer material above and below the point, respectively. However, the lateral thermal conductivity at the interface between layers of different materials is a weighted average of thermal conductivities for the materials above and below the point,

$$
K_{x y} \equiv \frac{\Delta z_{+} K_{x y+}+\Delta z_{-} K_{x y-}}{\Delta z_{+}+\Delta z_{-}} .
$$

For a 3D Cartesian coordinate system at each time step there are three tridiagonal matrix ADI update equations for each point in the cell space. These equations must explicitly include the $\Delta \mathrm{x}_{ \pm}$and $\Delta \mathrm{y}_{ \pm}$factors required to take into account the change in lateral cell size at the borders between rings in the extended boundaries. The appropriate equations are:

Substep 1:

$$
\begin{gathered}
T_{i, j, k+1}^{n+1 / 3}-\left(\frac{2 \Delta z \Delta z_{+}}{K_{z+}}\right)\left(\frac{C}{\Delta t}+\frac{K_{z+}}{2 \Delta z \Delta z_{+}}+\frac{K_{z-}}{2 \Delta z \Delta z_{-}}\right) T_{i, j, k}^{n+1 / 3} \\
+\left(\frac{\Delta z_{+} K_{z-}}{\Delta z_{-} K_{z+}}\right) T_{i, j, k-1}^{n+1 / 3} \\
=-\left(\frac{2 \Delta z \Delta z_{+} C}{\Delta t K_{z+}}\right) T_{i, j, k}^{n}-\left(\frac{2 \Delta z \Delta z_{+}}{K_{z+}}\right) g_{i, j, k}^{n} \\
-\left(\frac{2 \Delta z \Delta z_{+} K_{x}}{\Delta x \Delta x_{+} K_{z+}}\right)\left(T_{i+1, j, k}^{n}-T_{i, j, k}^{n}\right)
\end{gathered}
$$

$$
\begin{aligned}
& -\left(\frac{2 \Delta z \Delta z_{+} K_{x}}{\Delta x \Delta x_{-} K_{z+}}\right)\left(T_{i-1, j, k}^{n}-T_{i, j, k}^{n}\right) \\
& -\left(\frac{2 \Delta z \Delta z_{+} K_{y}}{\Delta y \Delta y_{+} K_{z+}}\right)\left(T_{i, j+1, k}^{n}-T_{i, j, k}^{n}\right) \\
& -\left(\frac{2 \Delta z \Delta z_{+} K_{y}}{\Delta y \Delta y_{-} K_{z+}}\right)\left(T_{i, j-1, k}^{n}-T_{i, j, k}^{n}\right) \\
& -\left(T_{i, j, k+1}^{n}-T_{i, j, k}^{n}\right)-\left(\frac{\Delta z_{+} K_{z-}}{\Delta z_{-} K_{z+}}\right)\left(T_{i, j, k-1}^{n}-T_{i, j, k}^{n}\right)
\end{aligned}
$$

$$
\begin{gathered}
=-\left(\frac{2 \Delta x \Delta x_{+} C}{\Delta t K_{x}}\right) T_{i, j, k}^{n+1 / 3}+\left(T_{i+1, j, k}^{n}-T_{i, j, k}^{n}\right) \\
+\left(\frac{\Delta x_{+}}{\Delta x_{-}}\right)\left(T_{i-1, j, k}^{n}-T_{i, j, k}^{n}\right)
\end{gathered}
$$

Substep 3:

$$
\begin{aligned}
T_{i, j+1, k}^{n+1}-( & \left.\frac{2 \Delta y \Delta y_{+}}{K_{y}}\right)\left(\frac{C}{\Delta t}+\frac{K_{y}}{2 \Delta y \Delta y_{+}}+\frac{K_{y}}{2 \Delta y \Delta y_{-}}\right) T_{i, j, k}^{n+1} \\
& +\left(\frac{\Delta y_{+}}{\Delta y_{-}}\right) T_{i, j-1, k}^{n+1} \\
= & -\left(\frac{2 \Delta y \Delta y_{+} C}{\Delta t K_{y}}\right) T_{i, j, k}^{n+2 / 3}+\left(T_{i, j+1, k}^{n}-T_{i, j, k}^{n}\right) \\
& +\left(\frac{\Delta y_{+}}{\Delta y_{-}}\right)\left(T_{i, j-1, k}^{n}-T_{i, j, k}^{n}\right)
\end{aligned}
$$

At the boundaries the equations have to be modified. One simple boundary condition is that of the perfect heat sink, $\mathrm{T}$ $=0$. This is easily implemented by replacing Eq. (17) for $\mathrm{i}=$ 0 and $i_{\max }$ and Eq. (18) for $\mathrm{j}=0$ and $\mathrm{j}_{\max }$ by

$T_{0, \text { max }}^{n+1}=0$.

The perfect insulator boundary condition is also easily implemented by the equations

$T_{0, \text { max }}^{n+1}-T_{1, \text { max }-1}^{n+1}=0$.

\section{MOVING FRAME OF REFERENCE}

In a reference frame that is moving at velocity $\mathbf{v}$ with respect to the heat source, the Fourier heat conduction equation is 


$$
\begin{gathered}
C(\overrightarrow{\mathbf{r}}, t)\left[\frac{\partial}{\partial t}-\overrightarrow{\mathbf{v}} \cdot \nabla\right] T(\overrightarrow{\mathbf{r}}, t)=\nabla \cdot[\tilde{\mathbf{K}}(\overrightarrow{\mathbf{r}}, t) \nabla T(\overrightarrow{\mathbf{r}}, t)] \\
+g(\overrightarrow{\mathbf{r}}, t)
\end{gathered}
$$

For motion of the film stack in the lateral plane relative to the incident optical spot, this equation reduces to

$$
\begin{aligned}
C(\overrightarrow{\mathbf{r}}, t)\left[\frac{\partial}{\partial t}-\mathrm{v}_{\mathrm{x}}\right. & \left.\frac{\partial}{\partial x}-\mathrm{v}_{\mathrm{y}} \frac{\partial}{\partial y}\right] T(\overrightarrow{\mathbf{r}}, t) \\
& =\nabla \cdot[\tilde{\mathbf{K}}(\overrightarrow{\mathbf{r}}, t) \nabla T(\overrightarrow{\mathbf{r}}, t)]+g(\overrightarrow{\mathbf{r}}, t)
\end{aligned} .
$$

The corresponding ADI CN equation is

$$
\begin{gathered}
C(\overrightarrow{\mathbf{r}}, t) \frac{\left[T^{n+1}(\overrightarrow{\mathbf{r}}, t)-T^{n}(\overrightarrow{\mathbf{r}}, t)\right]}{\Delta t}= \\
\left(\nabla \cdot \tilde{\mathbf{K}}(\overrightarrow{\mathbf{r}}, t) \nabla+C(\overrightarrow{\mathbf{r}}, t) \mathrm{v}_{\mathrm{x}} \frac{\partial}{\partial x}+C(\overrightarrow{\mathbf{r}}, t) \mathrm{v}_{\mathrm{y}} \frac{\partial}{\partial y}\right)^{.} \\
\cdot\left[\frac{T^{n+1}(\overrightarrow{\mathbf{r}}, t)+T^{n}(\overrightarrow{\mathbf{r}}, t)}{2}\right]+g(\overrightarrow{\mathbf{r}}, t)
\end{gathered}
$$

We propose the following three ADI equations for the Douglas-Gunn solution in the moving frame:

Substep 1:

$$
\begin{aligned}
T^{n+\frac{1}{3}}- & T^{n}=\left(\frac{K_{z} \Delta t}{C} \frac{\partial^{2}}{\partial z^{2}}\right)\left(\frac{T^{n+\frac{1}{3}}+T^{n}}{2}\right)+\left(\frac{K_{x} \Delta t}{C} \frac{\partial^{2}}{\partial x^{2}}\right) T^{n} \\
& +\left(\frac{K_{y} \Delta t}{C} \frac{\partial^{2}}{\partial y^{2}}\right) T^{n}+\left(\Delta t \mathrm{v}_{\mathrm{x}} \frac{\partial}{\partial x}\right) T^{n}+\left(\Delta t \mathrm{v}_{\mathrm{y}} \frac{\partial}{\partial y}\right) T^{n} \\
& +\frac{g \Delta t}{C}
\end{aligned}
$$

Substep 2:

$$
\begin{aligned}
T^{n+\frac{2}{3}}- & T^{n}=\left(\frac{K_{z} \Delta t}{C} \frac{\partial^{2}}{\partial z^{2}}\right)\left(\frac{T^{n+\frac{1}{3}}+T^{n}}{2}\right) \\
+ & \left(\frac{K_{x} \Delta t}{C} \frac{\partial^{2}}{\partial x^{2}}\right)\left(\frac{T^{n+\frac{2}{3}}+T^{n}}{2}\right)+\left(\frac{K_{y} \Delta t}{C} \frac{\partial^{2}}{\partial y^{2}}\right) T^{n} \\
& +\left(\Delta t \mathrm{v}_{\mathrm{x}} \frac{\partial}{\partial x}\right)\left(\frac{T^{n+\frac{2}{3}}+T^{n}}{2}\right)+\left(\Delta t \mathrm{v}_{\mathrm{y}} \frac{\partial}{\partial y}\right) T^{n}+\frac{g \Delta t}{C}
\end{aligned}
$$

Substep 3:

$$
\begin{aligned}
T^{n+1}- & T^{n}=\left(\frac{K_{z} \Delta t}{C} \frac{\partial^{2}}{\partial z^{2}}\right)\left(\frac{T^{n+\frac{1}{3}}+T^{n}}{2}\right) \\
+ & \left(\frac{K_{x} \Delta t}{C} \frac{\partial^{2}}{\partial x^{2}}\right)\left(\frac{T^{n+\frac{2}{3}}+T^{n}}{2}\right) \\
& +\left(\frac{K_{y} \Delta t}{C} \frac{\partial^{2}}{\partial y^{2}}\right)\left(\frac{T^{n+1}+T^{n}}{2}\right) \\
& +\left(\Delta t \mathrm{v}_{\mathrm{x}} \frac{\partial}{\partial x}\right)\left(\frac{T^{n+\frac{2}{3}}+T^{n}}{2}\right) \\
& +\left(\Delta t \mathrm{v}_{\mathrm{y}} \frac{\partial}{\partial y}\right)\left(\frac{T^{n+1}+T^{n}}{2}\right)+\frac{g \Delta t}{C}
\end{aligned}
$$

These equations can be simplified by subtracting Eq. (25) from Eq. (26), and Eq. (24) from Eq. (25). Combining these two new equations with Eq. (24) leads after some algebraic manipulation to the CN Eq. (23) to first order in $\Delta \mathrm{t}$.

The numerical implementation of the three tridiagonal ADI update equations is obtained from Eqs. (24) - (26) by substituting the numerical expansions for the Laplacian and gradient,

$\frac{\partial^{2} T}{\partial x^{2}} \cong \frac{T_{i+1}-2 T_{i}+T_{i-1}}{\Delta x^{2}}$

and

$\frac{\partial T}{\partial x} \cong \frac{T_{i+1}-T_{i-1}}{2 \cdot \Delta x}$.

Substep 1:

$$
\begin{aligned}
T_{i, j, k+1}^{n+\frac{1}{3}} & -2\left(1+\frac{\Delta z^{2} C}{K_{z} \Delta t}\right) T^{n+\frac{1}{3}}+T_{i, j, k-1}^{n+\frac{1}{3}} \\
=- & \left(\frac{2 \Delta z^{2} C}{K_{z} \Delta t}\right) T^{n}-\left(T_{i, j, k+1}^{n}-2 T_{i, j, k}^{n}+T_{i, j, k-1}^{n}\right) \\
& -\left(\frac{2 K_{x} \Delta z^{2}}{K_{z} \Delta x^{2}}\right)\left(T_{i+1, j, k}^{n}-2 T_{i, j, k}^{n}+T_{i-1, j, k}^{n}\right) \\
& -\left(\frac{2 K_{y} \Delta z^{2}}{K_{z} \Delta y^{2}}\right)\left(T_{i, j+1, k}^{n}-2 T_{i, j, k}^{n}+T_{i, j-1, k}^{n}\right) \\
& -\left(\frac{\Delta z^{2} C \mathrm{v}_{\mathrm{x}}}{K_{z} \Delta x}\right)\left(T_{i+1, j, k}^{n}-T_{i-1, j, k}^{n}\right) \\
& -\left(\frac{\Delta z^{2} C \mathrm{v}_{\mathrm{y}}}{K_{z} \Delta y}\right)\left(T_{i, j+1, k}^{n}-T_{i, j-1, k}^{n}\right)-\left(\frac{2 \Delta z^{2}}{K_{z}}\right) g
\end{aligned}
$$

Substep 2:

$$
\begin{aligned}
\left(1+\frac{C \mathrm{v}_{\mathrm{x}} \Delta x}{K_{x}}\right) T_{i+1, j, k}^{n+\frac{2}{3}}-2\left(1+\frac{C \Delta x^{2}}{K_{x} \Delta t}\right) T_{i, j, k}^{n+\frac{2}{3}} \\
+\left(1-\frac{C \mathrm{v}_{\mathrm{x}} \Delta x}{K_{x}}\right) T_{i-1, j, k}^{n+\frac{2}{3}} \\
=\left(1+\frac{C \mathrm{v}_{\mathrm{x}} \Delta x}{K_{x}}\right) T_{i+1, j, k}^{n}-2 T_{i, j, k}^{n} \\
+\left(1-\frac{C \mathrm{v}_{\mathrm{x}} \Delta x}{K_{x}}\right) T_{i-1, j, k}^{n}-\left(\frac{2 C \Delta x^{2}}{K_{x} \Delta t}\right) T_{i, j, k}^{n+\frac{1}{3}}
\end{aligned}
$$

Substep 3:

$$
\begin{aligned}
\left(1+\frac{C \mathrm{v}_{\mathrm{y}} \Delta y}{K_{y}}\right) T_{i, j+1, k}^{n+1}-2\left(1+\frac{C \Delta y^{2}}{K_{y} \Delta t}\right) T_{i, j, k}^{n+1} \\
+\left(1-\frac{C \mathrm{v}_{\mathrm{y}} \Delta y}{K_{y}}\right) T_{i, j-1, k}^{n+1} \\
=\left(1+\frac{C \mathrm{v}_{\mathrm{y}} \Delta y}{K_{y}}\right) T_{i, j+1, k}^{n}-2 T_{i, j, k}^{n} \\
\quad+\left(1-\frac{C \mathrm{v}_{\mathrm{y}} \Delta y}{K_{y}}\right) T_{i, j-1, k}^{n}-\left(\frac{2 C \Delta y^{2}}{K_{y} \Delta t}\right) T_{i, j, k}^{n+\frac{2}{3}}
\end{aligned}
$$

The unconditional stability of these equations is proven in Appendix 1. A simple example for an isotropic medium is considered in Appendix 2 for comparison to analytical results. In the next section the ADI algorithm with extended boundaries is applied to heat assisted magnetic recording. 


\section{EXAMPLES}

HAMR has been proposed for data storage densities of 1 $\mathrm{Tb} / \mathrm{in}^{2}$ or greater $[7,8]$. At these storage densities the optical spot size must be $\sim 50 \mathrm{~nm}$ or smaller and the spacing between the recording head and the recording medium must be as small as possible, typically $\leq 5 \mathrm{~nm}$, for adequate resolution of the bits in the readback signal. Both of these conditions are consistent with using near field optics to deliver the optical energy from the recording head to the recording medium. Unlike optical recording media for which a multilayer thin film stack is used to control both optical and thermal properties of the media, the film stack of a HAMR medium is designed primarily for its magnetic and thermal properties. The recording layer must be placed at the top of the film stack to minimize the head-to-media spacing. An aggressive heat sink must be laminated to the recording layer to ensure that thermal time constants on the order of $1 \mathrm{~ns}$ or less to achieve $\mathrm{GHz}$ data rates. A thermal spacer may be placed between the recording layer and the heat sink to further control this time constant. For the first example, we consider a three layer film stack consisting of $20 \mathrm{~nm}$ of a metallic recording material laminated to a $20 \mathrm{~nm}$ thermally insulating layer and a semi-infinite metallic heat sink as shown in Fig. (3). An optical source generates a circular optical spot with a Gaussian intensity profile which is absorbed in the top $5 \mathrm{~nm}$ layer of the recording medium, i.e.,

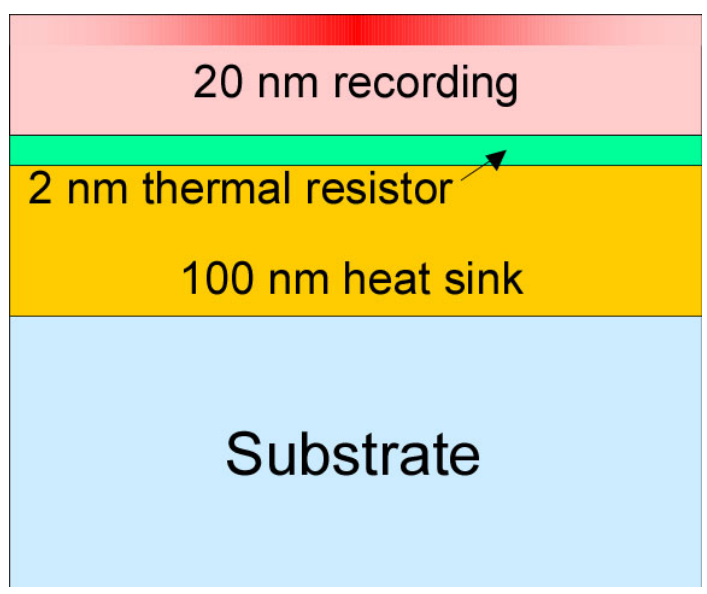

Fig. (3). Multilayer layer film stack for heat assisted magnetic recording.

$$
\begin{gathered}
I(x, y, z)=\left(2.0 \times 10^{18}\right) \exp \left(-\frac{x^{2}+y^{2}}{r^{2}}\right) \frac{W}{m^{3}} \\
\text { for } z<0
\end{gathered}
$$

where $r=50 \mathrm{~nm}$. The heat flow out of the top surface of the recording layer is assumed to be negligible. The thermal parameters are given in Table $\mathbf{1}$. The central region of fine discretization in each layer is $49 \times 49$ cells for the extended boundary calculation. This region of fine discretization is solved for both a $49 \times 49$ cell space and a $499 \times 499$ cell space with non-extended boundaries. Each cell has a lateral area of $(10 \mathrm{~nm})^{2}$. The calculation is carried out in the stationary frame of reference with time steps of 100 ps for 1000 steps. Each of these cases is computed for two different boundary conditions, the typical $\mathrm{T}=0$ boundary condition and the perfect insulator boundary condition, $\mathrm{dT} / \mathrm{dn}=0$. The six computed temperature profiles in the top layer of the metallic recording medium are plotted in Fig. (4).

Table 1. Thermal ADI Parameters for the Multilayer Medium in Fig. (3)

\begin{tabular}{|c|c|c|c|c|c|}
\hline Layer & Material & $\begin{array}{c}\# \mathbf{z} \\
\text { Cells }\end{array}$ & $\begin{array}{c}\mathbf{z} \text { Cell } \\
\text { Thickness }\end{array}$ & $\begin{array}{c}\mathbf{K} \\
\mathbf{W} /(\mathbf{K} \mathbf{~ m})\end{array}$ & $\begin{array}{c}\mathbf{C} \\
\times \mathbf{1 0}^{\mathbf{6}} \mathbf{J} /\left(\mathbf{m}^{\mathbf{3}} \mathbf{K}\right)\end{array}$ \\
\hline \hline 1 & recording & 4 & $5 \mathrm{~nm}$ & 100 & 3 \\
\hline 2 & dielectric & 2 & 10 & 1 & 3 \\
\hline 3 & heat sink & 5 & 100 & 400 & 3 \\
\hline 4 & substrate & 5 & 1000 & 400 & 3 \\
\hline 5 & substrate & 5 & 10000 & 400 & 3 \\
\hline
\end{tabular}

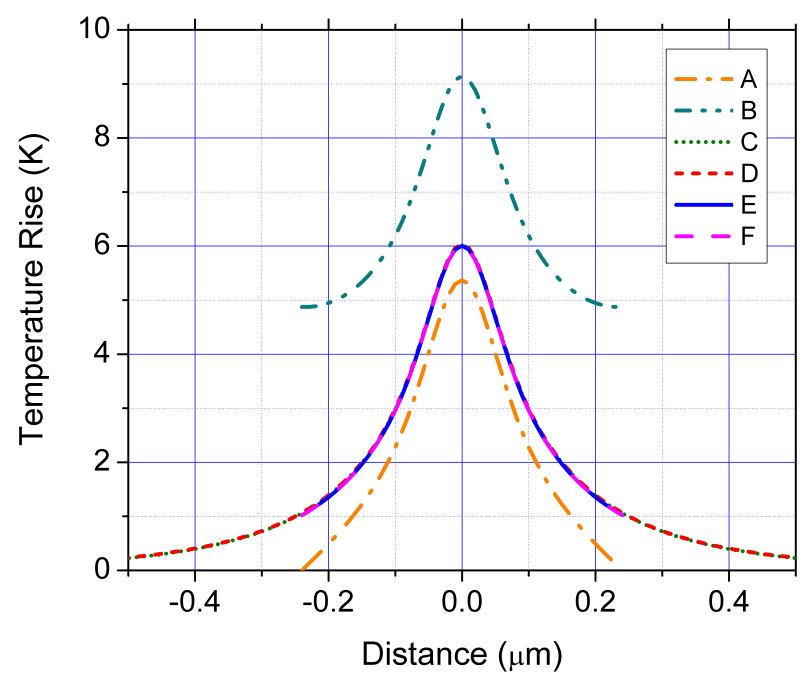

Fig. (4). Thermal profiles computed with and without the extended boundaries for different boundary conditions. The curves (A) - (D) are for non-extended boundaries, while (E) and (F) are for extended boundaries. (A), (B), (E) and (F) are for the smaller cell space, while (C) and (D) are for the larger cell space. (A), (C), and (E) are for $\mathrm{T}=0$ boundaries, while (B), (D), and (F) are for perfectly insulating boundaries.

The two calculations for the extended boundaries are essentially identical with each other and to the two calculations for non-extended boundaries using the larger cell space. In this case it is apparent that the boundaries are not influencing the calculations. On the other hand, the calculation for non-extended boundaries with $\mathrm{T}=0$ boundary conditions, curve (A), gives substantially lower temperatures, demonstrating that the boundaries are affecting the result by increasing the rate of heat flow to the outer surfaces. Likewise, the calculation with non-extended boundaries and $\mathrm{dT} / \mathrm{dn}=0$ boundary conditions, curve (B), is also significantly affected by the boundaries trapping heat in the system and causing larger temperatures than in the other calculations. Indeed, if the calculation is carried out to longer times, the temperature profile of this curve will continue to increase. For this example, accurate results are obtained for calculations $(\mathrm{C})-(\mathrm{F})$, but the results with the extended 
boundaries and smaller cell space are computed in about $2.4 \%$ of the time of those without the extended boundaries but much larger cell space.

Next we consider an example for a moving film stack. For current hard disc drives, the rotating disc corresponds to tangential media speeds of 10 to $50 \mathrm{~m} / \mathrm{s}$. The optical spot size is Gaussian with a $1 / \mathrm{e}$ radius of $25 \mathrm{~nm}$. The thin film stack is the same as the first example, and the total power absorbed by the medium is $1 \mathrm{~mW}$. We choose a cell space of $99 \times 99 \times 21$ cells and each cell has a lateral area of $(5 \mathrm{~nm})^{2}$. The calculation is carried out with time steps of $100 \mathrm{ps}$ for 1000 steps, which is much longer than necessary to reach the steady state response. The temperature profile in the center of the track at the top of the recording medium is computed in the down track direction for three different medium speeds in Fig. (5), and a contour plot of the thermal spot in the recording layer is shown in Fig. (6) for a speed of $50 \mathrm{~m} / \mathrm{s}$. The thermal bloom from the moving medium is relatively small, unlike the tear drop shape often found in optical storage media [5]. This indicates that the thermal time constant of the medium is fast compared to the motion of the medium with respect to the optical spot size. However, the thermal spot is relatively large compared to the incident optical spot and this limits the storage density of this medium.

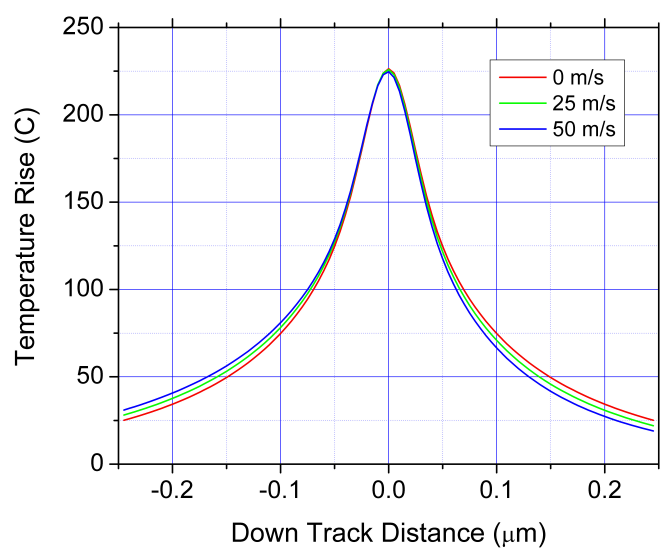

Fig. (5). Temperature profile along the track direction for HAMR medium at three different rotation speeds.

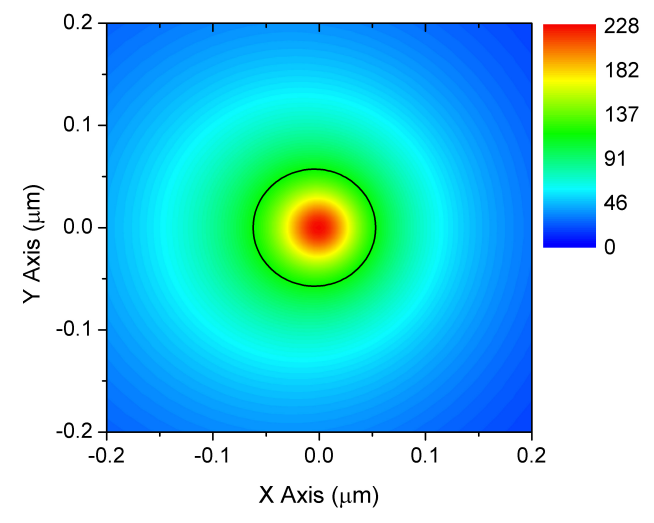

Fig. (6). Contour plot of the thermal spot in the recording layer for a rotation speed of $50 \mathrm{~m} / \mathrm{s}$.

\section{CONCLUSIONS}

A technique has been described for efficiently computing the thermal profile from an optical spot absorbed in a moving multilayer thin film stack in the near field. By making use of the moving frame of reference, the computation can be performed relatively quickly while still maintaining accurate boundary conditions. It is shown that a simple three layer medium can be designed for heat assisted magnetic recording with a relatively fast thermal time constant that is suitable for high data rates, but the thermal bloom from the optical spot is larger than desired for high storage densities.

\section{ACKNOWLEDGEMENTS}

We would like to acknowledge Hua Zhou, Julius Hohlfeld, Chubing Peng, Terry McDaniel, and Wei Peng for useful discussions. This work was performed as part of the INSIC HAMR ATP Program, with the support of the U.S. Department of Commerce, National Institute of Standards and Technology, Advanced Technology Program, Cooperative Agreement Number 70NANB1H3056.

\section{APPENDIX 1}

Stability of the Douglas-Gunn approach to the ADI equations has been discussed in Ref. [3]. We follow their approach and also simplify the notation with the following definitions.

$a_{u} \equiv \frac{1}{2} \Delta t \mathrm{v}_{u} \sin k_{u}$

and

$b_{u} \equiv \frac{C(\Delta u)^{2}}{K_{u} \Delta t}\left(1-\cos k_{u}\right)$

where $\mathrm{u}=\mathrm{x}, \mathrm{y}$, or $\mathrm{z}$.

We begin by taking the discrete spatial Fourier transform of Eqs. (29) - (31). If the discrete spatial Fourier transform of the temperature at point $(\mathrm{i}, \mathrm{j}, \mathrm{k})$ is defined for each wavevector

$k_{x}^{(m)}=\frac{2 \pi m}{N_{x}}, \quad m=0, \ldots ., N_{x}-1$

where $\mathrm{N}_{\mathrm{x}}$ is the number of cells along the $\mathrm{x}$ axis, as

$\mathfrak{I}\left(T_{i, j, k}\right) \equiv \hat{T}_{i, j, k}$

then

$\mathfrak{I}\left(T_{i+1, j, k}\right)=\left(\cos k_{x}+i \sin k_{x}\right) \hat{T}_{i, j, k}$

and

$\mathfrak{I}\left(T_{i-1, j, k}\right)=\left(\cos k_{x}-i \sin k_{x}\right) \hat{T}_{i, j, k}$.

The Fourier transforms of Eqs. (29) - (31) are

$\left(1+b_{z}\right) \hat{T}^{n+\frac{1}{3}}=\left(1-i a_{x}-i a_{y}-2 b_{x}-2 b_{y}-b_{z}\right) \hat{T}^{n}$ 
$\left(1+i a_{x}+b_{x}\right) \hat{T}^{n+\frac{2}{3}}=\hat{T}^{n+\frac{1}{3}}+\left(i a_{x}+b_{x}\right) \hat{T}^{n}$

and

$\left(1+i a_{y}+b_{y}\right) \hat{T}^{n+1}=\hat{T}^{n+\frac{2}{3}}+\left(i a_{y}+b_{y}\right) \hat{T}^{n}$.

Solving for $\hat{T}^{n+1}$ in terms of $\hat{T}^{n}$ gives

$\hat{T}^{n+1}=\frac{\left[\begin{array}{l}\left(\begin{array}{l}1-b_{x}-b_{y}-b_{z}-a_{x} a_{y}+b_{x} b_{y} \\ +b_{x} b_{z}+b_{y} b_{z}+b_{x} b_{y} b_{z}-a_{x} a_{y} b_{z}\end{array}\right) \\ +i\left(\begin{array}{l}a_{x} b_{y}+a_{y} b_{x}+a_{x} b_{z}+a_{y} b_{z} \\ +a_{x} b_{y} b_{z}+a_{y} b_{x} b_{z}\end{array}\right)\end{array}\right]}{\left(1+i a_{x}+b_{x}\right)\left(1+i a_{y}+b_{y}\right)\left(1+b_{z}\right)} \hat{T}^{n}$.

To further simplify the equations we can restrict our analysis without loss of generality to motion along the $\mathrm{x}$ axis, setting $\mathrm{a}_{\mathrm{y}}=0$. The stability criterion for the general update equation

$\hat{T}^{n+1}=(r+i s) \hat{T}^{n}$

is

$|r+i s|<1 \quad$ or $\quad r^{2}+s^{2}<1$.

Applying this criterion to Eq. (A10) gives

$\frac{-4\left(b_{x}+b_{y}+b_{z}\right)\left(1+b_{x} b_{y}+b_{x} b_{z}+b_{y} b_{z}+b_{x} b_{y} b_{z}\right)}{a_{x}^{2}\left(1+2 b_{y}+2 b_{z}+2 b_{y} b_{z}\right)}<1$

which is true for all values of $a_{x}$. Thus the moving frame 3D ADI equations are unconditionally stable.

\section{APPENDIX 2}

Consider an anisotropic medium with specific heat $\mathrm{C}$, and thermal conductivities $\mathrm{K}_{\mathrm{x}}, \mathrm{K}_{\mathrm{y}}$, and $\mathrm{K}_{\mathrm{z}}$. Let

$\alpha_{x}=\sqrt{\frac{C}{4 K_{x}}}$

$\alpha_{y}=\sqrt{\frac{C}{4 K_{y}}}$

$\alpha_{z}=\sqrt{\frac{C}{4 K_{z}}}$.

Consider a point source given by $q(x, y, z, t)=\delta(x) \delta(y) \delta(z) \delta(t)$. The temperature distribution generated by this source is

$$
g(x, y, z, t)=\frac{\Gamma}{t^{\frac{3}{2}}} \exp \left(-\frac{\alpha_{x}^{2} x^{2}+\alpha_{y}^{2} y^{2}+\alpha_{z}^{2} z^{2}}{t}\right)
$$

where

$\Gamma=\frac{8 \alpha_{x} \alpha_{y} \alpha_{z}}{(4 \pi)^{\frac{3}{2}} C}$.

The temperature distribution $\mathrm{G}(\mathrm{x}, \mathrm{y}, \mathrm{z}, \mathrm{t})$ for a Gaussian source $\mathrm{Q}(\mathrm{x}, \mathrm{y}, \mathrm{x}) \delta(\mathrm{t})$ where
$Q(x, y, z)=Q_{0} \exp \left(\frac{x^{2}+y^{2}+z^{2}}{r_{0}^{2}}\right)$

is

$$
\begin{aligned}
& G(x, y, z, t)=\int_{\tilde{x}=-\infty}^{\infty} \int_{\tilde{y}=-\infty}^{\infty} \int_{\tilde{z}=-\infty}^{\infty} \\
& Q(x-\tilde{x}, y-\tilde{y}, z-\tilde{z}) g(\tilde{x}, \tilde{y}, \tilde{z}, t) d \tilde{x} d \tilde{y} d \tilde{z} \\
& =\frac{Q_{0} \Gamma}{t^{\frac{3}{2}}} \int_{\tilde{x}=-\infty}^{\infty} \exp \left(-\frac{(x-\tilde{x})^{2}}{r_{0}^{2}}\right) \exp \left(-\frac{\alpha_{x}^{2} \tilde{x}^{2}}{t}\right) d \tilde{x} \\
& \cdot \int_{\tilde{y}=-\infty}^{\infty} \exp \left(-\frac{(y-\tilde{y})^{2}}{r_{0}^{2}}\right) \exp \left(-\frac{\alpha_{y}^{2} \tilde{y}^{2}}{t}\right) d \tilde{y} \\
& \cdot \int_{\tilde{z}=-\infty}^{\infty} \exp \left(-\frac{(z-\tilde{z})^{2}}{r_{0}^{2}}\right) \exp \left(-\frac{\alpha_{z}^{2} \tilde{z}^{2}}{t}\right) d \tilde{z} \\
& =Q_{0} \Gamma\left(\frac{\pi}{t}\right)^{\frac{3}{2}} \frac{\exp \left(-\frac{\alpha_{x}^{2} x^{2}}{t+\alpha_{x}^{2} r_{0}^{2}}\right)}{\sqrt{\left(\frac{\alpha_{x}^{2}}{t}+\frac{1}{r_{0}^{2}}\right)}} \\
& \frac{\exp \left(-\frac{\alpha_{y}^{2} y^{2}}{t+\alpha_{y}^{2} r_{0}^{2}}\right)}{\sqrt{\left(\frac{\alpha_{y}^{2}}{t}+\frac{1}{r_{0}^{2}}\right)}} \cdot \frac{\exp \left(-\frac{\alpha_{z}^{2} z^{2}}{t+\alpha_{z}^{2} r_{0}^{2}}\right)}{\sqrt{\left(\frac{\alpha_{z}^{2}}{t}+\frac{1}{r_{0}^{2}}\right)}}
\end{aligned}
$$

Now let the medium move rectilinearly with respect to the source with velocity $\mathrm{v}_{\mathrm{x}} \hat{\mathbf{x}}$ and let the Gaussian source be given by $\mathrm{Q}(\mathrm{x}, \mathrm{y}, \mathrm{z}) \mathrm{U}(\mathrm{t})$ in the reference frame attached to the source where $U(t)$ is the Heaviside unit step function. The temperature distribution at time $\mathrm{t}$ in the reference frame attached to the source is given by

$$
T(x, y, z, t)=\int_{\tau=0}^{t} G\left(x-\mathrm{v}_{\mathrm{x}} t+\mathrm{v}_{\mathrm{x}} \tau, y, z, t-\tau\right) d \tau .
$$

Thus,

$$
\begin{array}{r}
T(x, y, z, t)=\int_{\tau=0}^{t} Q_{0} \Gamma\left(\frac{\pi}{t-\tau}\right)^{\frac{3}{2}} \frac{\exp \left(-\frac{\alpha_{x}^{2}\left(x-\mathrm{v}_{x} t+\mathrm{v}_{x} \tau\right)^{2}}{t-\tau+\alpha_{x}^{2} r_{0}^{2}}\right)}{\sqrt{\left(\frac{\alpha_{x}^{2}}{t-\tau}+\frac{1}{r_{0}^{2}}\right)}} \\
\cdot \frac{\exp \left(-\frac{\alpha_{y}^{2} y^{2}}{t-\tau+\alpha_{y}^{2} r_{0}^{2}}\right) \exp \left(-\frac{\alpha_{z}^{2} z^{2}}{t-\tau+\alpha_{z}^{2} r_{0}^{2}}\right)}{\sqrt{\left(\frac{\alpha_{y}^{2}}{t-\tau}+\frac{1}{r_{0}^{2}}\right)\left(\frac{\alpha_{z}^{2}}{t-\tau}+\frac{1}{r_{0}^{2}}\right)}} d \tau
\end{array}
$$




$$
\begin{gathered}
=\int_{s=0}^{t} Q_{0} \Gamma\left(\frac{\pi}{s}\right)^{\frac{3}{2}} \frac{\exp \left(-\frac{\alpha_{x}^{2}\left(x-\mathrm{v}_{x} s\right)^{2}}{s+\alpha_{x}^{2} r_{0}^{2}}\right)}{\sqrt{\left(\frac{\alpha_{x}^{2}}{s}+\frac{1}{r_{0}^{2}}\right)}} . \\
\frac{\exp \left(-\frac{\alpha_{y}^{2} y^{2}}{s+\alpha_{y}^{2} r_{0}^{2}}\right) \exp \left(-\frac{\alpha_{z}^{2} z^{2}}{s+\alpha_{z}^{2} r_{0}^{2}}\right)}{\sqrt{\left(\frac{\alpha_{y}^{2}}{s}+\frac{1}{r_{0}^{2}}\right)\left(\frac{\alpha_{z}^{2}}{s}+\frac{1}{r_{0}^{2}}\right)}} d s
\end{gathered}
$$

We now compare this result to that obtained from the numerical ADI technique. Consider a Gaussian optical spot intensity profile with the functional dependence,

$$
I(x, y, z)=\left(1.0 \times 10^{18}\right) \exp \left(-\frac{x^{2}+y^{2}+z^{2}}{r^{2}}\right) \frac{W}{m^{3}} .
$$

for $\mathrm{z}<0$ and with $\mathrm{r}=100 \mathrm{~nm}$. The medium is moving at a speed of $10 \mathrm{~m} / \mathrm{s}$ relative to the optical spot. There is no heat flow out of the top surface. For a reference frame which is stationary with respect to the medium the central computation space must be rather long to include the entire distance traveled in the specified time interval. In a $1 \mu$ s interval the medium travels $10 \mu \mathrm{m}$. This can be accommodated by a stationary cell space for which the central region of fine discretization has $1000 \times 250 \times 57$ cells and for which each cell has a lateral area of $(20 \mathrm{~nm})^{2}$. The total length of the cell space in the central region along the $x$ axis is $20 \mu \mathrm{m}$ and if the optical spot is initially centered on the medium $5 \mu \mathrm{m}$ from one edge, after $1 \mu$ s the medium has moved so that the optical spot is centered $5 \mu \mathrm{m}$ from the other edge. The lateral extended boundaries are a millimeter from the center of the optical spot. The boundary at the bottom surface is only $100 \mu \mathrm{m}$ from the top surface, but this distance is found to be sufficient for accurate results with calculation times of up to $1 \mu$ s or more.

There are four layers with different vertical dimensions in the ADI calculations. The first layer consists of 30 cells, each with a vertical thickness of $10 \mathrm{~nm}$. The next layer has nine cells, each with a vertical thickness of $100 \mathrm{~nm}$. The third layer also has nine cells, each with a thickness of $1000 \mathrm{~nm}$. Finally the fourth layer has nine cells, each with a thickness of $10 \mu \mathrm{m}$. The total thickness of the film stack is thus about $100 \mu \mathrm{m}$. The thermal conductivity is equal to $1 \mathrm{~W} /(\mathrm{K} \mathrm{m})$ and the specific heat is $1 \times 10^{6} \mathrm{~J} /\left(\mathrm{m}^{3} \mathrm{~K}\right)$ for all cells. The time step is $1 \mathrm{~ns}$ and the calculations are carried out for 1000 time steps.

In the moving frame of reference the cell space can be made considerably smaller if one is only interested in the temperature profile in the vicinity of the optical spot. For this example the calculation in the moving frame of reference has a central cell space of $200 \times 200 \times 57$ cells. The extended lateral boundaries are again a millimeter from the center of the optical spot. As can be seen in Fig. (7), there is excellent agree- ment between the stationary and moving frame ADI results and the analytical result. The calculation for the moving frame was more than five times faster than that of the stationary frame, although of course the thermal profile is computed over a much smaller cell space. This result demonstrates the validity of both the extended boundaries and the ADI equations for the moving frame of reference.

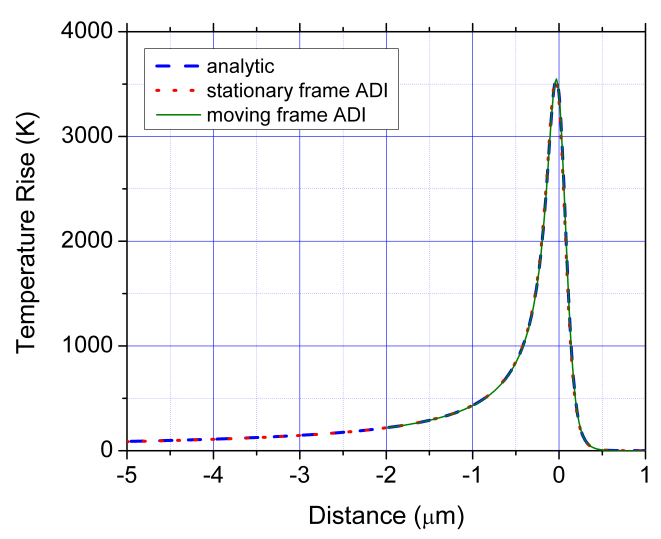

Fig. (7). Comparison of the temperature rise after $1 \mu$ s for a Gaussian optical spot on a half space of isotropic media for the analytic and the extended boundary ADI calculations in both the moving and stationary frames.

\section{REFERENCES}

[1] Peaceman DW, Rachford HH. The Numerical Solution of Parabolic and Elliptic Differential Equations. J Soc Indust Appl Math 1955; 3: $28-41$.

[2] Özişik MN. Boundary Value Problems of Heat Conduction. New York: Dover; 1968; Sect. 9.7.

[3] Wang T-Y, Chen CC-P. 3-D Thermal-ADI: A Linear-Time Chip Level Transient Thermal Simulator. IEEE Trans Comp-Aided Des Integ Circ Syst 2002; 21: 1434-1445.

[4] Mansuripur M, Connell GAN, Goodman JW. Laser induced heating of multilayers. Appl Opt 1982; 21: 1106-1114.

[5] Mansuripur M, Connell GAN. Laser induced heating of moving multilayer media. Appl Opt 1983; 22: 666-670.

[6] Peng C, Cheng L, Mansuripur M. Experimental and theoretical investigations of laser induced crystallization and amorphization in phasechange optical recording media. J Appl Phys 1997; 82: 4183-4191.

[7] Rausch T, Mihalcea C, Pelhos K, et al. Near Field Heat Assisted Magnetic Recording with a Planar Solid Immersion Lens. Jpn J Appl Phys 2006; 45: 1314-1320.

[8] McDaniel TW, Challener WA, Sendur K. Issues in Heat-Assisted Perpendicular Recording. IEEE Trans Magn 2003; 39: 1972-1979.

[9] Douglas J, Gunn JE. A general formulation of alternating direction methods Part I. Parabolic and hyperbolic problems. Numer Math 1964; 6: 428-453.

[10] Itagi A. Virtual Optical Recording System, Ph.D. Thesis, Carnegie Mellon University; 2003.

[11] Itagi A. Finite Volume Method for the Fourier Heat Conduction in Layered Media with a Moving Volume Heat Source. Jpn J Appl Phys 2007; 46: 1482-1489. 